\title{
Impact of maraviroc on immune restoration in an advanced stage HIV-infected patient
}

\author{
Sylvie Bregigeon ${ }^{1}$, Amélie Menard ${ }^{1}$, Olivia Faucher ${ }^{1}$, Catherine Tamalet ${ }^{2}$, Caroline Solas ${ }^{3}$, Véronique Obry-Roguet ${ }^{1}$, \\ Isabelle Poizot-Martin ${ }^{1 *}$
}

From $16^{\text {th }}$ International Symposium on HIV and Emerging Infectious Diseases

Marseille, France. 24-26 March 2010

\section{Background}

Maraviroc is a CCR5 antagonist with clearly demonstrated virological efficacy in patients refractory to prior treatment and infected with an R5-tropic virus.

This antiretroviral drug would appear to have a special immunomodulatory property, given the significantly higher increase in CD4 count in patients treated with maraviroc in clinical trials.

We report the case of a severely immunocompromised patient in which the introduction of maraviroc reduced viral load to below the threshold of 40 copies $/ \mathrm{ml}$, with an increase in CD4 of over 100 in the space of 17 months.

\section{Methods}

The patient was a 53 year old man diagnosed with HIV in 1987, and classed stage C according to CDC classification for cerebral toxoplasmosis in June 1995. His history includes cerebral histoplasmosis in April 1997 (relapse in April 2006) and stage IV Hodgkin's lymphoma with visceral involvement in October 2006 (CD4 $\left.=1 / \mathrm{mm}^{3}\right)$. The first antiretroviral treatment was given in October $1995\left(\mathrm{CD} 4<50 / \mathrm{mm}^{3}\right)$, and the first maraviroc-based combination therapy was his $28^{\text {th }}$ line treatment (16 stoppages for treatment failure). In January 2008, the tropism test (trofile test) gave an R5 profile and the genotypic resistance test (ANRS algorithm July 2009) showed, for the RT gene, resistance to AZT, 3TC, D4T, ddI, ABC, EFV, NVR and possibly to TDF and ETV; for the protease gene, resistance to IDVr, SQVr, NFV, FPVr, ATV, DRVr, LPVr and possibly to TPVr.

\section{Results}

Table 1 shows viro-immunological changes with treatment:
Table 1

\begin{tabular}{lllllll}
\hline & D0 & M3 & M6 & M12 & M15 & M17 \\
\hline VLlog/ml & 3.83 & 2.63 & 5.68 & 1.6 & 1.6 & 1.6 \\
CD4/mm & 82 & 138 & 132 & 147 & 207 & 200 \\
CD4\% & 11.4 & 13.5 & 13.1 & 12.2 & 15.1 & 15.1 \\
CD8/mm & 346 & 445 & 524 & 596 & 661 & 575 \\
CD8\% & 48.3 & 43.7 & 51.7 & 49.5 & 48.3 & 43.5 \\
MVC add & FTC/RAL/ETV & TPVr/ETV & TVD/DRVr/ETV \\
to & 1 month & 5 months & 12 months & & & \\
\hline
\end{tabular}

\section{Discussion}

This case report shows the possibility of being confronted with an R5-tropic virus after 21 years' progression, and 15 years of antiretroviral treatment, with severe immunosuppression. The three successive maraviroc-based combination therapies lead to an increase in CD4 count independent of the virological response, with a delta of +120 CD4 after 17 months. A parallel increase in the CD8 count was also observed, as has been reported in clinical trials.

\section{Author details}

${ }^{1}$ Department of Immuno-hematology, HIV-Clinical center, Hospital SainteMarguerite, Marseilles, France. ${ }^{2}$ Fédération de Microbiologie Clinique, Hôpital de la Timone, Marseilles, France. ${ }^{3}$ Laboratoire de Pharmacocinétique et de Toxicologie, Hôpital de La Timone, Marseilles, France.

Published: 11 May 2010

doi:10.1186/1742-4690-7-S1-P59

Cite this article as: Bregigeon et al:: Impact of maraviroc on immune restoration in an advanced stage HIV-infected patient. Retrovirology 2010 7(Suppl 1):P59.

* Correspondence: isabelle.poizot@mail.ap-hm.fr

'Department of Immuno-hematology, HIV-Clinical center, Hospital

SainteMarguerite, Marseilles, France 